Bryant University

Bryant Digital Repository

Mathematics Department Journal Articles

Mathematics Department Faculty Publications and Research

1974

\title{
A Model for the Response of Seated Humans to Sinusoidal Displacements of the Seat
}

Robert Muksian

Bryant University

Charles D. Nash

University of Rhode Island

Follow this and additional works at: https://digitalcommons.bryant.edu/math_jou

\section{Recommended Citation}

Muksian, Robert and Nash, Charles D., "A Model for the Response of Seated Humans to Sinusoidal Displacements of the Seat" (1974). Mathematics Department Journal Articles. Paper 46.

https://digitalcommons.bryant.edu/math_jou/46

This Article is brought to you for free and open access by the Mathematics Department Faculty Publications and Research at Bryant Digital Repository. It has been accepted for inclusion in Mathematics Department Journal Articles by an authorized administrator of Bryant Digital Repository. For more information, please contact dcommons@bryant.edu. 


\title{
A MODEL FOR THE RESPONSE OF SEATED HUMANS TO SINUSOIDAL DISPLACEMENTS OF THE SEAT*
}

\author{
ROBERT MUKSIAN \\ Mathematics Department, Bryant College, Smithfield, Rhode Island 02917, U.S.A. \\ and \\ Charles D. Nash, JR.
}

Department of Mechanical Engineering and Applied Mechanics, University of Rhode Island, Kingston, Rhode Isiand 02881, U.S.A.

\begin{abstract}
A lumped-parameter model of the human body in the sitting position is formulated which includes the head, vertebral column, upper torso, abdomen-thorax viscera, pelvis and legs. The differential equations of motion for the rigid-body representation of the isolated masses were written for nonlinear springs and dashpots which represented the elasticity and damping, respectively, of the physical system. Also included in the equations of motion were coulomb friction forces for modeling sliding surfaces and related muscle contractions in the body, and the ballistocardiographic and diaphragm muscle forces. With all motions restricted to the vertical direction and sinusoidal displacements of the seat as the inputs, excellent agreement with mechanical and subjective experimental responses was achieved. Additionally, in agreement with the frequency dependency of muscle forces, the possibility of frequency dependent damping coefficients was implied.
\end{abstract}

\section{INTRODUCTION}

With the imminence of high speed mass transportation, the need to predict human response to continuous and impact type displacements while seated remains important relative to the areas of fatigue and injury. Extensive experimental work has been performed investigating head to seat and shoulder to seat acceleration ratios, driving point impedances, and subjective responses, such as pain and discomfort, for human beings in the sitting position. Theoretical models, both continuous and lumped-parameter, with varying degrees of complexity have been formulated in efforts to predict responses to specified inputs. The lumped-parameter models usually have been single or multidegree-of-freedom systems with linear, passive elements, whereas the human body is neither a passive nor a linear system. Carmichael (1968) states: "... the body is a nonlinear system with respect to the equations of motion...." Wittmann and Phillips (1969) conclude: "... existing nonlinearities are sufficient to be of concern... and that appropriate measures to determine the effect of these nonlinearities should be taken". Perng (1970) states: "The most striking feature of the mechanical behavior of biological tissues ... the stressstrain relation is remarkably nonlinear...". Markolf and Steidel (1970) state: "It is known that joint stiffnesses increase with deformation...". Belytschko et al.

* Received 9 Nowember 1973.

B.M. -Vol. 7. Not, ; B
(1972) refer to but neglect nonlinearities stating: “... because of the difficulties of characterizing a nonlinear, orthotropic composite material." When representing nonlinear elements of the body, the assumption of linear springs and dashpots may be too great a simplification for some inputs.

This paper reports the results of an investigation of a human body in the sitting position represented by a multidegree-of-freedom, lumped-parameter model which differs from previous models in that it more nearly describes the anatomical paths (spinal and visceral) between the pelvis and head; it includes active forces within the body; and it includes nonlinear, passive elements. The inputs to the model are limited to longitudinal (vertical when sitting) sinusoidal displacements of the seat, and the responses of the model are limited to motions in the vertical direction only.

\section{EQUATIONS OF MOTION}

An anatomical description of the human body in the sitting position was formulated by isolating the joints in the body where movements are allowed. According to Gray's Anatomy (1966), these include the vertebral column from the occipital bone of the skull to the sacrum; the attachments of the ribs to the vertebrae and to the sternum; the attachments of the sacrum to the pelvis; the attachments of the clavicle and scapula; and the abdomen-thorax viscera. Depending upon the selection of organs within the abdominal cavity, this 
description of the human body may be represented by 65-70 rigid bodies coupled with nonlinear springs and dashpots wherein are lumped the stiffness and damping qualities of the viscera, muscles, intervertebral discs, ligaments, fascia, etc.

The complexities of the structure, anatomical description, and mathematical model of the human body necessitated a simplification which is shown in Fig. 1 and presented by Muksian (1970). The following assumptions (with justifications) are made to formulate the model of this paper.

(1) The head and atlas (because of the condyloid joints connecting them) are combined into a single rigid body called the 'head' and labeled $m_{1}$.

(2) Since measurement of the relative motion between the vertebrae is not perfected for longitudinal excitation of live subjects, the remainder of the vertebral column (axis through sacrum) is combined into a single rigid body called the 'back' and labeled $m_{2}$.

(3) For deformations less than those that cause rupture of the intervertebral discs, experimental evidence (Pradko, 1966, 1967) indicates that the stiffness and damping in the vertebral column are represented by linear springs and linear dashpots, respectively labeled $K_{p 1}$ and $C_{p 1}$ for the cervical section and $K_{p 2}$ and $C_{p 2}$ for the thoracic and lumbar sections, for the transmission of the longitudinal components of the forces in the intervertebral discs, the longitudinal ligaments, and the attached muscles. In addition, pelvic stiffness and damping are included. For the linear elements, $p$ is assigned a value of unity.

(4) Since the relative motion between the ribs,

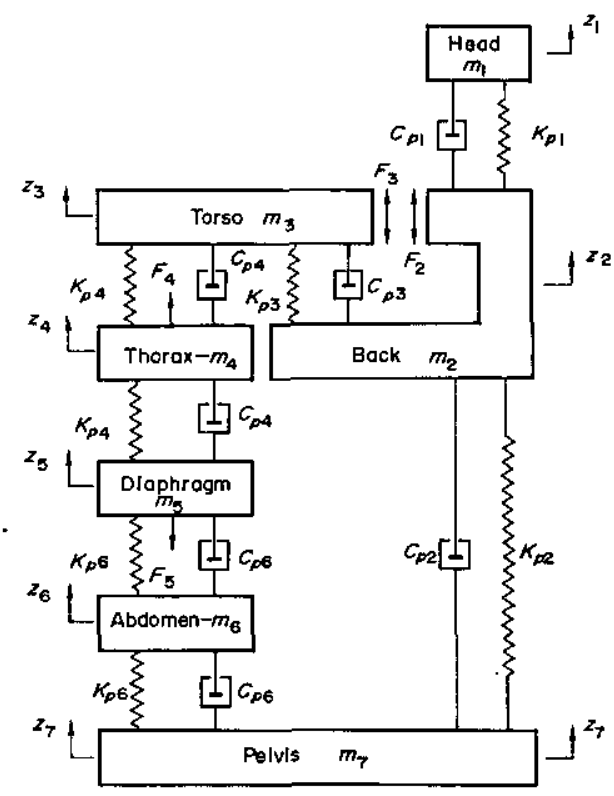

Fig. 1. The model. between the ribs and thoracic vertebrae, and between the costal cartilages and the sternum appears to be relatively slight and, in fact, does not appear to have been measured for longitudinal excitation of live subjects, the thoracic cage is combined into a single body called the 'torso' and labeled. $m_{3}$. Included in the torso are the arms and shoulders.

(5) Torso stiffness and damping are represented by a. nonlinear spring and a nonlinear dashpot, respectively labeled $K_{p 3}$ and $C_{p 3}$, for the transmission of the longitudinal components of the forces in the ligaments that attach the ribs to the vertebrae. In addition, a sliding surface between the torso and back, labeled $F_{2}$ and $F_{3}$, represents the longitudinal components of the forces in the gliding joints between the ribs and vertebrae as well as muscle contractions due to the relative velocities of ribs and vertebrae at the joints.

(6) The viscera of the thorax and abdomen are considered to be three rigid bodies called the 'thorax' (heart and lungs), 'diaphragm' (respiratory diaphragm), and 'abdomen' (liver, stomach, kidneys, spleen, gall and urinary bladders, pancreas, intestines) and labeled $m_{4}, m_{5}$ and $m_{6}$, respectively, with the stiffiness and damping characteristics of the thorax and abdomen represented by nonlinear springs and nonlinear dashpots. These are labeled $K_{p 4}$ and $C_{p 4}$ for the thorax and $K_{p t}$ and $C_{p 6}$ for the abdomen.

(7) The ballistocardiographic force $\left(F_{4}\right)$ acts on the thorax and the diaphragm muscle force $\left(F_{5}\right)$ acts on the diaphragm.

(8) The remainder of the body (pelvis and legs) is combined and called 'pelvis' and labeled $m_{7}$.

(9) Since joint stiffness increases with deformation (Markolf and Steidel, 1970), the springs are considered to be hard and the dashpots are nonlinear in the same manner as the hard springs. For the nonlinear elements, $p$ is assigned values of 1 and 3 .

(10) The heartbeat frequency increases linearly with the frequency of external excitation to the pelvis, and beyond approx. $10 \mathrm{~Hz}$, the heart rate returns to an almost normal value (Hood et al., 1966; von Gierke, 1970). The magnitude of the maximum value of the ballistocardiographic force is directly proportional to the heartbeat frequency.

(11) Respiration rate is proportional to the heartbeat frequency (Ruch and Fulton, 1960), and the diaphragm muscle force varies linearly with respiration rate.

With Newton's second law considered to be axiomatic, free-body-diagrams of the rigid bodies in Fig. 1 give equations of motion about the vertical equilibrium positions in the form

$$
m_{i} z_{i}^{\prime \prime}=\sum F_{i}(t)-\sum_{j} D_{j i}-\sum_{j} S_{j i} \quad i=1,2, \ldots, 7
$$


where $j$ is summed over the number of springs or dashpots attached to the $i$ th mass.

Since spring and viscous damping forces are due to relative displacements and velocities, respectively, between two coupled bodies, forces in cubic (hard) springs are given by

$$
S_{j i}=\sum_{p} K_{p j}\left(z_{i}-z_{n}\right)^{p} \quad p=1,3
$$

where $z_{n}$ represents the displacements of the mass above (if any) and the mass below (if any) the ith mass, and forces in cubic dashpots are given by

$$
D_{j i}=\sum_{p} C_{p j}\left(z_{i}^{\prime}-z_{n}^{\prime}\right)^{g} \quad p=1,3
$$

where $z_{n}^{\prime}$ represents the velocities of the mass above (if any) and the mass below (if any) the $i$ th mass. The coulomb friction forces between the back and torso are given by signum functions in the forms

$$
F_{i}=\left|F_{i}\right| \operatorname{Sgn}\left(z_{i+1}^{\prime}-z_{i}^{\prime}\right)
$$

and

$$
F_{i+1}=\left|F_{i+1}\right| \operatorname{Sgn}\left(z_{i}^{\prime}-z_{i+1}^{\prime}\right)
$$

where the absolute value notation is used to represent the magnitude of the force.

Substitution of equations (2-5) into equation (1) and rearranging gives the differential equations of motion

$$
\begin{aligned}
m_{i} z_{i}^{\prime \prime}+\sum_{j} \sum_{p} C_{p j}\left(z_{i}^{\prime}-z_{n}^{\prime}\right)^{p} & +\sum_{j} \sum_{p} K_{p j}\left(z_{i}-z_{n}\right)^{p} \\
& =\Sigma F_{i}(t) . \quad i=1,2, \ldots, 7 .
\end{aligned}
$$

For a sinusoidal displacement input of the seat $\left(m_{7}\right)$ given by

$$
z_{7}=z_{7 m} \operatorname{Sin}\left(2 \Pi f_{7} t\right),
$$

where $z_{7 m}$ is the maximum input displacement, the system of equations (6) is reduced by one, since equation (7) gives an imposed solution to $z_{7}^{\prime \prime}$.

In order to specify the input acceleration, $z_{7}^{\prime \prime}$, in terms of body wt, $G$ 's, the maximum displacement is specified in the form

$$
z_{7 m}=\frac{G g}{\left(2 \Pi f_{7}\right)^{2}}
$$

Then $z_{7}$ and $z_{7}^{\prime}$ in equations (6) are specified by

$$
z_{7}=\frac{G g}{\left(2 \Pi f_{7}\right)^{2}} \operatorname{Sin}\left(2 \Pi f_{7} t\right)
$$

and

$$
z_{7}^{\prime}=\frac{G g}{2 \Pi f_{7}} \operatorname{Cos}\left(2 \Pi f_{7} t\right) .
$$

Since analytical solutions to the class of nonlinear differential equations of the model have not been dis- covered to date, numerical solutions were necessary. Using the conventional transformation, velocity was defined by

$$
z_{i}^{\prime}=v_{i}
$$

transforming acceleration to

$$
z_{i}^{\prime \prime}=v_{i}^{\prime} .
$$

Substituting equations (11 and 12)-into equations (6) and rearranging gives

$$
\begin{array}{r}
v_{i}^{\prime}=-\frac{1}{m_{j}}\left\{\sum_{j} \sum_{p} C_{p j}\left(v_{i}-v_{n}\right)^{p}+\sum_{j} \sum_{p} K_{p j}\left(z_{i}-z_{n}\right)^{p}\right. \\
\left.-\sum F_{i}(t)\right\} \quad i=1,2, \ldots, 6
\end{array}
$$

which is the algorithm for the numerical solutions. Equations (13) were programmed for solution on the IBM System/360 Model 50 digital computer using the Runge-Kutta method (with Gill's coefficients) as the numerical procedure.

\section{MODEL DATA}

Although their effects may be minor, the ballistocardiographic and diaphragm muscle forces were included in the model.

Based on approximations to Ruch and Fulton (1960), who show the general shape of the pressure differential between the left ventricle of the heart and the aorta, Hood et al. (1966), comments by von Gierke (1970) and Miller (1970), the ballistocardiographic force was included as a sinusoidal pulse with a duration of oneeighth the heartbeat period as shown in Fig. 2, and with a maximum value given as a function of thorax frequency, $f_{4}$, in the form

$$
F_{4 m}=0.024 f_{4} \mathrm{lb}
$$

with $f_{4}$ given by

$$
\left.\begin{array}{rr}
f_{4}=\frac{2 \cdot 5 f_{7}+75}{60} \mathrm{~Hz} & 0 \leq f_{7} \leq 10 \\
f_{4}=\frac{-4 f_{7}+140}{60} \mathrm{~Hz} & 10<f_{7} \leq 15 \\
f_{4}=1.33 \mathrm{~Hz} & f_{7}>15
\end{array}\right\} .
$$

Ruch and Fulton (1960) show that the minimum work of respiration occurs at a respiration rate of approximately $30 \mathrm{cycles} / \mathrm{min}$, and as respiration rate increases due to activity, the respiration work increases. The diaphragm, which is one of the major muscles for respiration, was assumed to have a frequency which is one-half the heartbeat frequency. Thus,

$$
f_{5}=\frac{1}{2} f_{4} \mathrm{~Hz}
$$




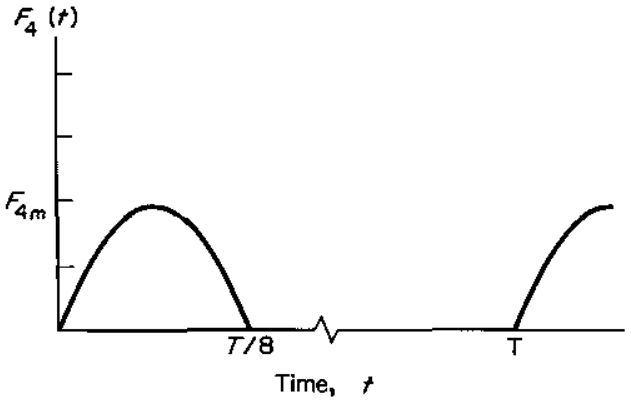

Fig. 2. The ballistocardiographic force $-F_{4}(t)$.

The diaphragm muscle force, which appears very difficult to measure, was included as a linear function of the diaphragm frequency in the form

$$
F_{5}=1+c f_{5} \mathrm{lb}
$$

where the constant, $c$, has units of $\mathrm{lb} / \mathrm{Hz}$ and was assigned a value of unity. The force is assumed to have a duration of one-half the heartbeat period as shown in Fig. 3.

A total subject wt of $176 \mathrm{lb}$ was used for the model with a distribution of weight, shown in Table 1 , which was approximated from Hertzberg and Clauser (1964). Colella (1969) and Roberts et al. (1969). According to Hertzberg and Clauser (1964), this weight constitutes the 80 th percentile of 4060 U.S. Air Force flying personnel.

The estimate of the spring stiffness for the vertebral column, shown in Table 2, is in excellent agreement with the experimental data of Vogt et al. (1968). Since there is a lack of information concerning the stiffness of the abdomen-thorax viscera, an assumed value was used. The damping coefficient of the vertebral column was estimated from Coermann et al. (1960) and Vogt et al. (1968) using a damping ratio of 0.6 and a singledegree-of-freedom system. The damping coefficients of the abdomen-thorax viscera were assumed. In order to assess the sensitivity of the model to erroneous

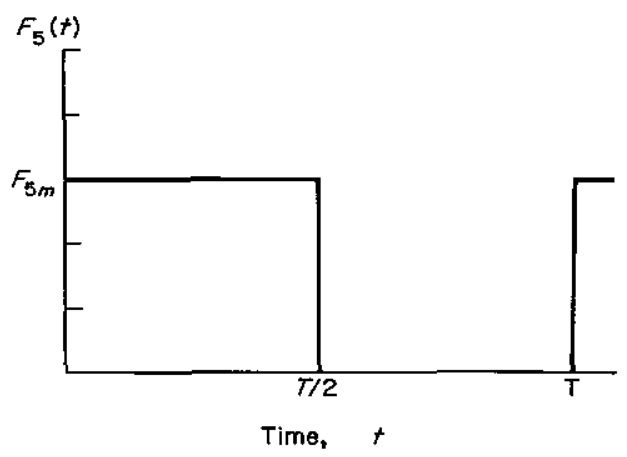

Fig. 3. The diaphragm muscle force- $F_{5}(t)$.
Table 1. Weight distribution of an average human male

\begin{tabular}{lcc}
\hline Element & Weight (lb) & Mass (slugs) \\
\hline Head & 12 & 0.372 \\
Back & 15 & 0.466 \\
Torso & 72 & $2 \cdot 234$ \\
Thorax & 3 & 0.093 \\
Diaphragm & 1 & $0 \cdot 031$ \\
Abdomen & 13 & $0 \cdot 404$ \\
Pelvis & 60 & 1.861 \\
Totals & 176 & $5 \cdot 461$ \\
\hline
\end{tabular}

The 'torso' includes the weight of the shoulder girdle and arms, and the 'pelvis' includes the weight of the legs.

Table 2. Estimated spring and dashpot coefficients

\begin{tabular}{crrcc}
\hline No. $(j)$ & $K_{1 j}$ & $K_{3 j}$ & $C_{1 j}$ & \multicolumn{1}{c}{$C_{3 j}$} \\
\hline 1 & 3600 & 0 & $245(370)$ & 0 \\
2 & 3600 & 0 & $245(370)$ & 0 \\
3 & 3600 & 3600 & $245(370)$ & $245(370)$ \\
4 & 60 & 60 & $20(30)$ & $20(30)$ \\
6 & 60 & 60 & $20(30)$ & $20(30)$ \\
\hline
\end{tabular}

The units for the coefficients are as follows: $K_{15}-\mathrm{lb} / \mathrm{ft}$; $K_{3 j}-\mathrm{lb} / \mathrm{ft}^{3} ; C_{1 j}-\mathrm{lb} /(\mathrm{sec} / \mathrm{ft}) ; C_{3 j}-\mathrm{lb} /(\mathrm{sec} / \mathrm{ft})^{3}$.

assumptions of the damping coefficients, the equations of motion were re-solved using values of damping coefficients approx 50 per cent greater than the original estimates. In this manner, we expect that we have probably bracketed the true values of the damping coefficients. The spring stiffnesses and damping coefficients are summarized in Table 2.

The coulomb friction forces $\left(F_{2}\right.$ and $\left.F_{3}\right)$ were assumed to equal the thoracic cage weight of $72 \mathrm{lb}$ and were held constant throughout the investigation.

The frequency of the sinusoidal input, $f_{7}$, was varied from 1 to $30 \mathrm{~Hz}$ in $1 \mathrm{~Hz}$ increments, and the maximum input acceleration was held constant at $1 \mathrm{~g}$. The maximum input displacement, $z_{7 \mathrm{~m}}$, varied from $0.816 \mathrm{ft}$ for $f_{7}=1 \mathrm{~Hz}$ to $0.00091 \mathrm{ft}$ for $f_{7}=30 \mathrm{~Hz}$ as determined from equation (8). The computer was programmed to terminate the calculations when the displacement of the head reached steady-state, increment the input frequency, and restart the calculations from the equilibrium position.

\section{RESULTS}

Figure 4 shows the curve, labeled Low $C_{p j}$, of the predicted head to seat (pelvis) acceleration ratio $(A R)$ as a function of seat frequency. Superimposed are the experimental values given by Goldman and von Gierke (1960), where the driving profile is not given except that the inputs are sinusoidal vibrations, and Pradko 


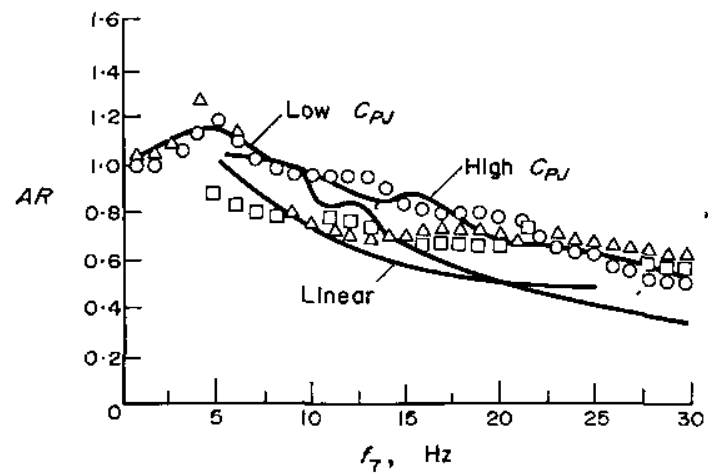

Fig. 4. Head to seat acceleration ratio-AR. $\mathrm{O}$, Goldman (1960) Sinusoidal vibrations. $\triangle$, Pradko $(1966,1967)$ Sinusoidal vibrations. $\square$, Pradko (1967) Random vibrations $-10 \mathrm{~Hz}$ BPF.

et al. $(1966,1967)$, whose experimental data show the responses to both random and sinusoidal inputs, where the average of the sinusoidal input acceleration levels over the frequency range of this paper is approx $1 \mathrm{~g}$. The low frequency predictions for sinusoidal inputs are generally within 5 per cent. At $12 \mathrm{~Hz}$, the curve is in the middle of the experimental data being 17 per cent higher than one set of data and 17 per cent lower than the other set. Between 1 and $9 \mathrm{~Hz}$, the model predictions and the data are in close agreement. Between 10 and $15 \mathrm{~Hz}$, the curve is closer to the data of Pradko et al. $(1966,1967)$, but it has the shape of the data of Goldman and von Gierke (1960). Beyond $15 \mathrm{~Hz}$, the curve diverges from converging data.

The curve, labeled High $C_{p j}$, shows the predicted. head to seat acceleration ratio for the higher damping coefficients used to gage the sensitivity of the model to that parameter. The two curves show that the model predicts excellent agreement with Goldman and von Gierke (1960) if the originally estimated damping coef-' ficients are used for input frequencies up to approx

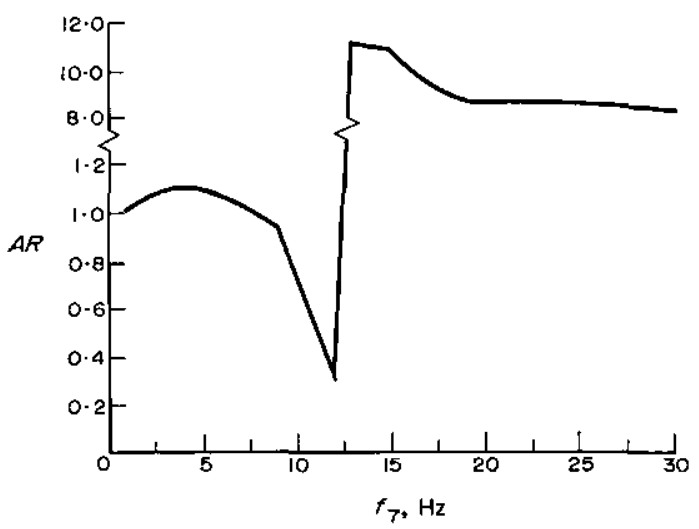

Fig. 5. Back to seat acceleration ratio- $A R$.
$8 \mathrm{~Hz}$ and the higher damping coefficients are used beyond $8 \mathrm{~Hz}$. Because of this, computations for the High $C_{p j}$ curve were not extended below $5 \mathrm{~Hz}$. The implication of a frequency dependent damping coefficient may be supported by Ruch and Fulton (1960) who show that, beyond $5 \mathrm{~Hz}$, the response force in a muscle which is subjected to an external stimulus is dependent upon the frequency of the stimulus.

Justification for the inclusion of nonlinear effects in the model of Fig. 1 was established by solving the equations of motion for the linear version of this model over a frequency range from 5 to $25 \mathrm{~Hz}$ with the same driving profile as for the nonlinear version. This curve, labeled linear, is also shown in Fig. 4. The computations for the linear model were not extended below $5 \mathrm{~Hz}$ because the results shown in Fig. 4 clearly indicate the inadequacy of the model.

Figure 5 shows a discontinuity in the predicted back to seat acceleration ratio for input frequencies between 9 and $13 \mathrm{~Hz}$ inclusive. Figure 6 shows a severe discontinuity at $12 \mathrm{~Hz}$ for the predicted torso to seat acceleration ratio. For input frequencies beyond $12 \mathrm{~Hz}$, the phase angle between the torso and, seat exceeds $90^{\circ}$.

$A R$

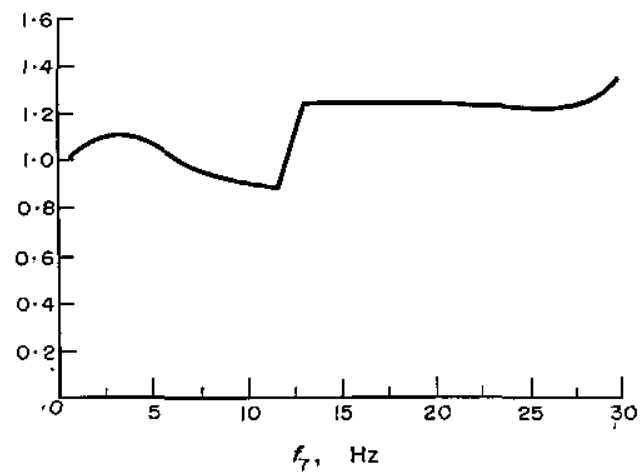

Fig. 6. Torso to seat acceleration ratio- $-A R$.

Figures $7-9$ show the predicted acceleration ratios for the thorax, the diaphragm, and the abdomen. The first resonant peak for each of the curves occurs at approx $3 \mathrm{~Hz}$ in general agreement with the results of Coermann et al. (1960) and Roberts et al. (1966) in a review of the results of others. In addition, the curves show four of the model's six resonant peaks.

In agreement with results cited by Roberts et al. (1966), the superposition, shown in Fig. 10, of the curves of the acceleration ratios of the thorax, diaphragm, and abdomen show the predicted motions of the three masses are approximately the same. Also included in Fig. 10 are the curves of the acceleration ratios of the head, back, and torso for input frequencies 


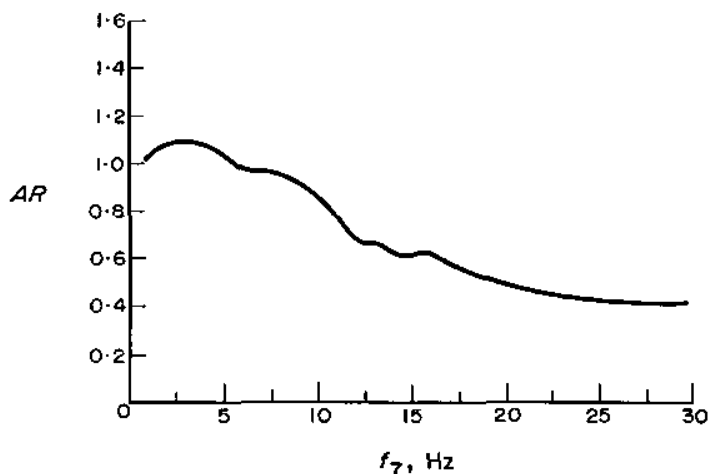

Fig. 7. Thorax to seat acceleration ratio- $A R$.

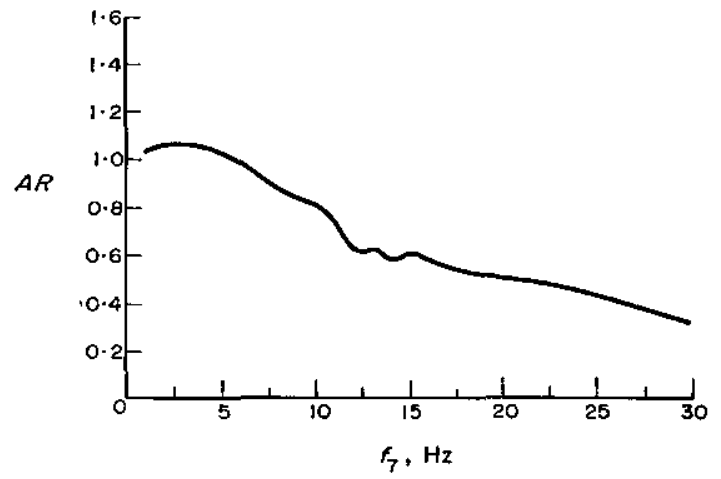

Fig. 8. Diaphragm to seat acceleration ratio- $-A R$.

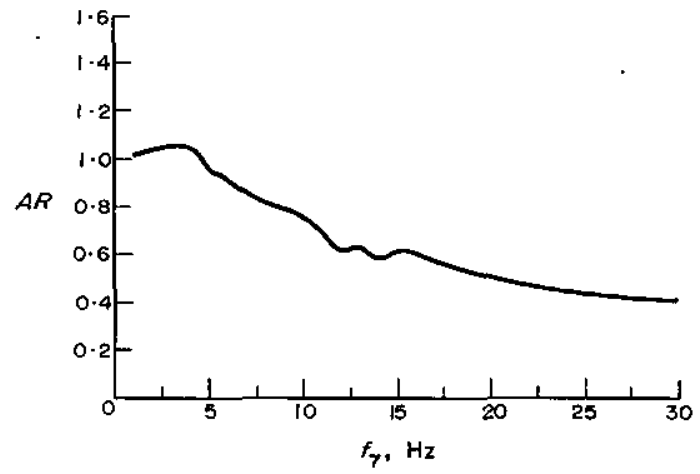

Fig. 9. Abdomen to seat acceleration ratio- $A R$.

less than $10 \mathrm{~Hz}$. The maximum spread between the six curves is approx 20 per cent which occurs between approx 6 and $8 \mathrm{~Hz}$ input frequencies. The proximity of the six curves in the low frequency range indicates that the model prediction is that the body moves as a single mass at these input frequencies and is in agreement with Coermann et al. (1960) and the results. cited by Roberts et al. (1966).

To assess the accuracy of the model, its predictions

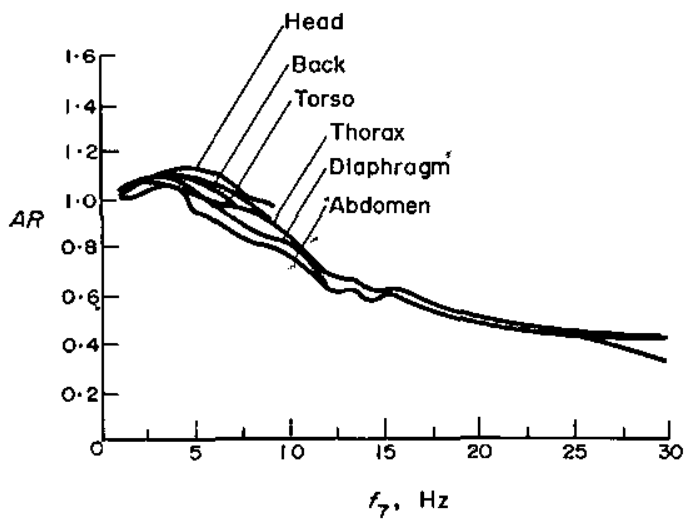

Fig. 10. Superposition of acceleration ratio curves.

for acceleration ratios and resonant frequencies have been compared with quantitative experimental data of other investigators. Another, and perhaps equally effective, correlation is the subjective response of live subjects to sinusoidal, longitudinal vibrations. Magid et al. (1962) presents such qualitative data. For comparable inputs, two input frequency ranges with a transition region were recorded. In the low frequency range, the number of responses for 'back pain' increased for input frequencies up to 6 or $7 \mathrm{~Hz}$ which is the order of magnitude $(4-5 \mathrm{~Hz})$ predicted by the model. The model discontinuities, as shown in Figs. 5 and 6, predict a transition region between 9 and $12 \mathrm{~Hz}$ which compares favorably with a transition region for subjective responses between 9 and $11 \mathrm{~Hz}$. Between 5 and $9 \mathrm{~Hz}$ input frequéncies, voluntary muscle contractions with severe intensities were recorded by Magid et al. (1962), and in the high input frequency range, severe intensities of muscle tone (involuntary) were recorded between 13 and $20 \mathrm{~Hz}$. The discontinuities of the model responses appear to predict these frequency ranges.

\section{CONCLUSIONS}

Based on the agreement between predictions and both quantitative and qualitative experimental data, the nonlinear model of this investigation represents, with acceptable tolerances, a human body in the sitting position which is subjected to vertical vibrations.

The predicted transition frequency range, as indicated by the model discontinuities, and the implied frequency dependent damping coefficients require further study.

\section{REFERENCES}

Belytschko, T., Schultz, A. B., Kulak, R. F. and Galante, J. O. (1972) Numerical stress analysis of intervertebral disk. ASME Paper No. 72-WA/BHF-12. 
Carmichael, J. B., Jr. (1968) Asst. Chief, Vibrations and Impact Branch, Biodynamics and Bionics Division, WrightPatterson AFB, Ohio. Correspondence.

Coermann, R. R., Ziegenruecker, G. H., Wittwer, A. L. and von Gierke, H. E. (1960) The passive dynamic mechanical properties of the human thorax-abdomen system and of the whole body system. J. Aero. Med. 31, 443-455.

Colella, A. (1969) Pathologist, State of Rhode Island. Correspondence regarding autopsy findings and procedures. Fogarty Memorial Hospital, Woonsocket.

Goldman, D. E. and von Gierke, H. E. (1960) The effects of shock and vibration on Man. Naval Med. Research Inst. Rpt. No. 60-3, Bethesda.

Gray, H. (1966) In Anatomy of the Human Body (Edited by Goss, C. M.) 28th Edn. Lea \& Febiger, Philadelphia.

Hertzberg, H. T. E. and Clauser, C. (1964) Size and motion. Bioastronautic Data Book, NASA SP-3006, pp. 241-271, NASA, Washington.

Hood, W. B., Jr., Murray, R. H., Urschel, C. W., Bowers, J. A. and Clark, J. G. (1966) Cardiopulmonary effects of whole-body vibration in man. J. appl. Physiol. 21, 17251731.

Magid, E. B., Coermann, R. R., Lowry, R. D. and Bosley, W. J. (1962) Physiological and mechanical response of the human to longitudinal whole-body vibration as deter. mined by subjective response. Wright-Patterson AFB Rpt. No. MRL-TDR-62-66.

Markolf, K. L. and Steidel, R. F., Jr. (1970) The dynamic characteristics of the human intervertebral joint. ASME Paper No. 70-WA/BHF-6.

Miller, H. (1970) Cardiologist. Unpublished data. Providence, Rhode Island.

Muksian, R. (1970) A nonlinear model of the human body in the sitting position subjected to sinusoidal displacements of the seat. Ph.D. Dissertation, University of Rhode Island. Kingston.

Perng, F. G. (1970) Strain energy function for biological tissue. ASME Paper No. 70-BHF-11.

Pradko, F., Lee, R. and Kaluza, V. (1966) Theory of human vibration response. ASME Paper No. 66-WA/BHF-15.

Pradko, F., Lee, R. and Greene, J. D. (1967) Human Vibration Response Theory. ASME Biomechanics Monograph, pp. 205-222.
Roberts, S. B., Ward, C. C. and Nahum, A. M. (1969) Head trauma-A parametric dynamic study. ASME Paper No. 69-BHF-11.

Roberts, V. L., Stech, E. L. and Terry, C. T. (1966) Review of mathematical models which describe human response to acceleration. ASME Paper No. 66-WA/BHF-13.

Ruch, T. C. and Fulton, J. F. (Editors). (1960) In Medical Physiology and Biophysics, 18th Edn. Saunders, Philadelphia.

Vogt, H. L., Coermann, R. R. and Fust, H. D. (1968) Mechanical impedance of the sitting human under sustained acceleration. J. Aero. Med. 39, 675-679.

Von Gierke, H. E. (1970) Telephone communication with C. D. Nash to Wright-Patterson AFB, Ohio.

Wittman, T. J. and Phillips, N. S. (1969) Human body nonlinearity and mechanical impedance analyses. J. Biomechanics 2, 281-288.

\section{NOMENCLATURE}

$C_{p j} \quad$ viscous damping coefficient, $\mathrm{lb}-(\mathrm{sec} / \mathrm{ft})^{p}$

$D_{j l} \quad$ viscous damping force on the $i$ th mass due to the $j$ th dashpot, $\mathrm{lb}$

$F_{i} \quad$ external force on the $i$ th mass except spring and dashpot forces, $\mathrm{lb}$

$f_{i} \quad$ frequency associated with the ith mass, $\mathrm{Hz}$

$G \quad$ number of body wts

$g$ acceleration due to gravity, $\mathrm{ft} / \mathrm{sec}^{2}$

$i \quad$ subscript representing a particular mass

$j$ subscript representing a particular spring or dashpot

$K_{p j} \quad$ spring stiffness, lb/(ft $)^{p}$

$m_{i} \quad$ mass of the $i$ th rigid body, $\mathrm{lb}-\mathrm{sec}^{2} / \mathrm{ft}$

$p \quad$ exponent on relative displacements and relative velocities for nonlinear springs and dashpots, respectively

$S_{j i} \quad$ spring force on the ith mass due to the $j$ th spring, Ib

$t$ time, sec

period, sec/cycle

velocity of the $i$ th mass, $\mathrm{ft} / \mathrm{sec}$

displacement of the $i$ th mass, $\mathrm{ft}$

first derivative with respect to time second derivative with respect to time $3 \cdot 14159$. 